
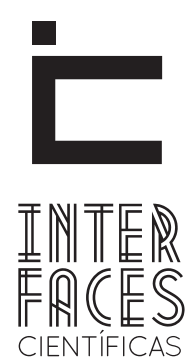

SAÚDE E AMBIENTE

ISSN IMPRESSO 2316-3313

E - ISSN 2316-3798

DOI - 10.17564/2316-3798.2017v5n3p67-76

\title{
O IMPACTO DA HOSPITALIZAÇÃO NA FUNCIONALIDADE E NA FORÇA MUSCULAR APÓS INTERNAMENTO EM UNIDADE DE TERAPIA INTENSIVA
}

\author{
THE IMPACT OF HOSPITALIZATION ON MUSCULAR FUNCTIONALITY AND STRENGTH AFTER INTENSIVE THERAPY UNITS \\ EL IMPACTO DE LA HOSPITALIZACIÓN EN LA FUNCIONALIDAD Y EN LA FUERZA MUSCULAR DESPUÉS DE LA INTERNACIÓN EN UNIDADE \\ DE TERAPIA INTENSIVA
}

Ana Carolina do Nascimento Calles ${ }^{1}$

Camila de Menezes Almeida ${ }^{3}$

Lara dos Santos Camilo ${ }^{5}$
Afrânio Torres de Oliveira Junior ${ }^{2}$ Evelin Aparecida Batista de Oliveira ${ }^{4}$

\section{RESUMO}

O longo período de internação em Unidade de Terapia Intensiva (UTI) pode gerar prejuízos em diversos sistemas, tais como o sistema musculoesquelético, cardíaco, respiratório e neurológico. Após a alta da UTI os pacientes apresentam debilidades que podem persistir por até um ano, incapacitando-os a realizar as suas Atividades de Vida Diárias (AVD's). Este trabalho teve por objetivo avaliar a variação da força muscular periférica e funcionalidade em pacientes hospitalizados. Estudo prospectivo, com- posto por 32 pacientes conscientes na UTI, aplicando-se o Medical Research Council (MRC) e o Índice de Barthel por um mesmo avaliador no momento da admissão e na alta da UTI. Apesar da média geral do MRC ter sido reduzida na alta, a força muscular periférica não sofreu alterações significativas, entretanto a funcionalidade apresentou diminuição significativa no domínio "atividades rotineiras", como higiene pessoal, cuidados com o rosto, cabelo, dentes e barba. Não houve perda significativa da 
força muscular periférica, entretanto, ocorreu uma redução da funcionalidade apresentada pelos pacientes no momento da alta.

\section{ABSTRACT}

A long period of internment in an Intensive Care Unit (ICU) can lead to losses in various body systems as the musculoskeletal, heart, respiratory and neurological. After discharge from the ICU patients present weaknesses that can persist for up to one year, disabling them to make their Daily Life Activities (ADLs). This study aimed to evaluate the variation of peripheral muscle strength and function in hospitalized patients. Prospective study, with 32 aware patients in ICU, where were applied the Medical Research Council (MRC) and the Barthel Index by the same examiner when the patient get in the ICU and on the moment of his discharge. Despite the MRC's

\section{RESUMEN}

El largo período de permanencia en la Unidad de Cuidados Intensivos $(\mathrm{UCI})$ puede conducir a pérdidas en diversos sistemas, tales como el sistema músculo-esquelético, cardíaco, respiratorio y nervioso. Después del alta de la $\mathrm{UCl}$, pacientes presentan debilidades que pueden persistir hasta por un año, los incapacita para realizar sus actividades de la vida diaria (AVD). Este estudio tuvo como objetivo evaluar la variación de la fuerza muscular periférica y la funcionalidad en pacientes hospitalizados. Estudio prospectivo con 32 pacientes conscientes en la UCl, aplicando el Medical Research Council (MRC) y el Índice de Barthel por el mismo examinador al ingreso y al alta. Aunque el promedio general

\section{PALAVRAS-CHAVE}

Força muscular, Unidade de terapia intensiva, Funcionalidade.

overall average was reduced in high, peripheral muscle strength did not change significantly, but the functionality has significantly decreased in the field "routine activities", like the personal hygiene, care with the face, hair, teeth and beard. There was no significant loss of peripheral muscle strength, however, despite a slight drop functionality presented by patients at discharge.

\section{KEYWORDS}

Muscle strength, Intensive Care Unit, Functionality.

del MRC se redujo en alta, la fuerza muscular periférica no cambió significativamente, sin embargo, la funcionalidad se redujo significativamente en el campo "actividades de rutina", tales como la higiene personal, cuidado de la cara, el pelo, los dientes y la barba. No hubo pérdida significativa de la fuerza muscular periférica, sin embargo, hubo una reducción de la funcionalidad presentada por los pacientes al alta.

\section{PALABRAS CLAVE}

Fuerza muscular, Unidad de Cuidados Intensivos, Funcionalidad 


\section{INTRODUÇ̃̃̃O}

Um dos desfechos avaliados após a alta da Unidade de Terapia Intensiva (UTI) é a independência funcional com o objetivo de avaliar e quantificar as alterações apresentadas pelos pacientes após a alta hospitalar. São poucos os estudos que avaliam esses desfechos em longo prazo, sendo necessário melhor entendimento sobre a repercussão funcional destes indivíduos resultante da internação hospitalar (SACANELLA et al., 2011; KUWABARA et al., 2013).

0 imobilismo no leito hospitalar, comum nos pacientes críticos acomete diversos sistemas, tais como o sistema musculoesquelético, cardiorrespiratório, metabólico e neurológico. 0 excesso de descanso e inatividade muscular decorrente do desuso dos membros ou do corpo promove ao músculo um declínio de massa muscular, levando a uma diminuição da força e endurance muscular (RIVOREDO; MEIJA, 2013).

De acordo com o exposto por Sibinelli e outros autores (2012), o sistema musculoesquelético deve se manter em movimento. São necessários apenas sete dias de repouso no leito hospitalar para reduzir a força muscular em $30 \%$, com perda adicional de $20 \%$ da força restante a cada semana que se passa. 0 desenvolvimento da fraqueza muscular generalizada é uma complicação frequente e significante nos pacientes admitidos em UTI, com incidência de 30 a 60\%. Múltiplos fatores podem contribuir para ocorrência desta condição, dentre eles destaca-se a imobilidade prolongada (SILVA; MAYNARD; CRUZ, 2010).

$O$ paciente que se encontra em UTI apresenta restrições motoras graves, incapacitando-os de realizar suas AVD's em decorrência da fraqueza muscular, fadiga constante e declínio funcional. A mobilização precoce e o posicionamento adequado no leito são indicados e essenciais como meio de reabilitação e interação do paciente com o ambiente e devem ser considerados como estímulo sensório-motor, prevenindo complicações secundárias ao imobilismo (FELICIANO et al., 2012).

Entretanto, a incidência de complicações decorrentes dos efeitos da imobilidade na UTI, contribui para o declínio funcional, aumento dos custos assistenciais, redução da QV e sobrevida pós-alta. Com o objetivo de atender a esta nova demanda as UTI's do Brasil e do mundo, cada vez mais buscam novas alternativas na resolução deste problema. A fisioterapia, capaz de promover a recuperação e preservação da funcionalidade por meio do movimento humano e suas variáveis, enquadramse como destaque nesta nova perspectiva (AzEREDO, 1984; BUHOP, 1996; CIESLA, 1996; STILLER, 2000; MALKOÇ; KARADIBAK; YILDIRIM, 2009; DESAI, 2011).

Assim, a intervenção precoce é fundamental e de extrema importância para que possíveis danos não se instalem, além da redução dos efeitos decorrentes do imobilismo, como melhora da função cardiorrespiratória, musculoesquelética, melhora do nível de consciência, aumento da independência funcional e aumento da qualidade de vida. 0 tratamento do fisioterapeuta deve ser iniciado dessa forma, para que o risco de hospitalização prolongada seja evitado e assim a imobilização, sendo um dos fatores primordiais para a recuperação do paciente (MOTA; SILVA, 2012).

De acordo com o exposto, o imobilismo acarreta a perda e diminuição da força muscular nos pacientes admitidos na UTI, interferindo de modo direto em sua funcionalidade. Sendo assim, o presente estudo justifica-se em verificar a perda de força muscular periférica pelo MRC, e a independência funcional e mobilidade por meio do Índice de Barthel, colaborando ou não com o que foi descrito na literatura analisada, revertendo casos de dependência ou incapacidade.

O objetivo do trabalho foi avaliar a força muscular periférica e funcionalidade em pacientes hospitalizados e sua variação em comparação com a admissão na UTI e alta da mesma.

\section{MÉTODO}

Trata-se de um estudo prospectivo, realizado no Hospital Vida, localizado na cidade de Maceió-AL. Somente foi iniciada a coleta de dados após aprovação do Comitê de Ética e Pesquisa (CEP), em conformidade com a resolução 466/12, com número do 
CAEE: 45667915.2.0000.5641. Todos os voluntários e/ou responsáveis pelos pacientes da pesquisa foram esclarecidos sobre o propósito do projeto e assinaram o Termo de Consentimento Livre e Esclarecido (TCLE).

Tratou-se de uma amostra não probabilística por conveniência, composta por pacientes conscientes internos na UTI, no período de agosto a outubro de 2015. O recrutamento dos pacientes foi realizado por um mesmo pesquisador que visitou diariamente o setor da UTI do Hospital Vida, selecionando, por meio de uma lista de checagem do próprio hospital, os pacientes que se enquadraram no perfil da população do estudo.

Foram incluídos na pesquisa os pacientes em tratamento na UTI com nível de consciência para realizar o Medical Research Council (MRC) e o Índice de Barthel, tanto na admissão, quanto na alta hospitalar. Foram adotados como critérios para exclusão da pesquisa: os pacientes que apresentam alterações no nível de consciência, avaliado por meio de um diálogo com os pacientes, no cognitivo, limitações físicas e pacientes que foram a óbito durante o internamento.

Todos os pacientes foram submetidos a avaliação que consistiu em dados pessoais, exame clínico e físico antes de iniciar a aplicação do teste de força muscular periférica (MRC) e avaliação da funcionalidade pelo Índice de Barthel. Um único avaliador realizou a avaliação da força muscular periférica e Índice de Barthel.

Foi realizado o MRC na admissão dos pacientes na UTI referente ao momento atual e na alta para a enfermaria, utilizado constantemente por ser de fácil execução e baixo custo. Sendo o grau de força muscular medida nos quatro membros (ombros, cotovelos, punhos, quadris, joelhos e tornozelos). Para isso, os mesmos se mantiveram em decúbito dorsal no leito, simétricos e era solicitada a realização dos movimentos com acréscimo da resistência manual aplicada pelo avaliador por todo movimento articular. A pontuação é dada pelo escore do Conselho de Pesquisas Médicas (CPM), com valores variando de 0 (nenhuma contração visível) a 60 (força muscular normal). Pacientes com o escore menor que 48 são considerados como portadores de fraqueza muscular, e acima de 49 , força normal (DE JONGHE et al., 2007).

A independência funcional foi avaliada por meio do Índice de Barthel, com os seguintes domínios: alimentação, banho, atividades rotineiras (como higiene pessoal, cuidados com o rosto, cabelo, dentes e barba), vestir-se, intestino, sistema urinário, uso do toalete, transferências, mobilidade e escadas. Pacientes com escore de 0 a 15 apresentam dependência total, 20 a 35 dependência grave, 40 a 55 dependência moderada, 60 a 95 dependência leve e 100 independência. Na admissão hospitalar, os pacientes eram avaliados na UTI, em relação ao período pré-hospitalar, e reavaliados na enfermaria de acordo com o momento da alta (MCDOWELL; NEWELL, 1996).

Os dados foram tabulados em banco de dados pelo programa EXCEL, Windows 8 e posteriormente analisados no programa SPSS versão 20.0.0s dados categóricos estão apresentados como frequência relativa e absoluta, já os dados contínuos (idade e dias de internação) estão como média e desvio-padrão. Os dados referentes ao MRC e o Barthel estão apresentados como Mediana, Média e valores mínimos e máximos. A comparação antes e depois desses dados foi realizada por meio do teste não-paramétrico de Wilcoxon. Para comparar as categorias dicotômicas de MRC (fraqueza $x$ normal) antes e depois, foi utilizado o teste de McNemar. Para todas as análises um valor de alfa igual a $5 \%$ foi adotado.

\section{RESULTADOS}

A amostra foi composta por 32 pacientes que preencheram os critérios de inclusão. Na Tabela 1, verificam-se variáveis como o sexo e os motivos de internação na UTI. Observa-se maior prevalência do sexo masculino e doença renal como principal motivo da internação. 
Tabela 1 - Caracterização da amostra estudada $(n=32)$

\begin{tabular}{|l|l|l|}
\hline Variável & N & $\%$ \\
\hline Sexo & & \\
\hline Masculino & 18 & 56,3 \\
\hline Feminino & 14 & 43,8 \\
\hline Motivo da Internação & & \\
\hline Doença Renal & 11 & 34,4 \\
\hline Doença Cardiovascular & 5 & 15,6 \\
\hline Infecção & 5 & 15,6 \\
\hline Doença Gastrointestinal & 2 & 6,3 \\
\hline Outro & 9 & 28,1 \\
\hline
\end{tabular}

Fonte: Dados da pesquisa.

A Tabela 2 apresenta dados da média de idade e tempo médio de internação.

Tabela 2 - Média da idade e tempo de internação apresentado pelos pacientes

\begin{tabular}{|l|l|l|}
\hline Variável & Média & DP \\
\hline Idade (anos) & 54,4 & 19,5 \\
\hline Tempo de internação (dias) & 5,3 & 2,7 \\
\hline
\end{tabular}

DP - Desvio-padrão.

Fonte: Dados da pesquisa.

A Tabela 3 compara os valores obtidos pelo MRC na admissão e na alta dos pacientes, subdivididos em MRC total, força dos membros superiores e membros inferiores.

Conforme apresentado, não houve diferença significativa entre os valores no momento da admissão e na alta dos indivíduos.
Tabela 3 - Comparação antes e depois dos valores de MRC dos pacientes estudados

\begin{tabular}{|l|l|l|l|l|l|l|l|}
\hline \multirow{2}{*}{ Variável } & \multicolumn{2}{|l|}{ Admissão } & \multicolumn{2}{l|}{ Alta } & \multirow{2}{*}{ * } \\
\cline { 2 - 7 } & Mediana & Média & Mín-Máx & Mediana & Média & Mín-Máx & \\
\hline MRC & 59 & 54,2 & $34-60$ & 60 & 54,9 & $34-60$ & 0,39 \\
\hline $\begin{array}{l}\text { MRC } \\
\text { Superior }\end{array}$ & 30 & 28,2 & $20-30$ & 30 & 28,5 & $20-30$ & 0,38 \\
\hline $\begin{array}{l}\text { MRC } \\
\text { Inferior }\end{array}$ & 29 & 26,0 & $10-30$ & 30 & 26,5 & $10-30$ & 0,30 \\
\hline
\end{tabular}

Legenda: MRC: Medical Research Council;

Mín: mínimo; Máx: máximo;

*Adotando $p<0,05$ para diferença estatística, com teste de Wilcoxon;

Fonte: Dados da pesquisa.

De acordo com o exposto na Tabela 4, houve um aumento no número de pacientes que apresentaram força muscular normal, quando comparado o momento da admissão e da alta, porém não houve diferença estatística.

Tabela 4 - Categoria MRC, quanto ao número de pacientes com fraqueza muscular e força normal

\begin{tabular}{|l|l|l|l|l|l|}
\hline \multirow{2}{*}{$\begin{array}{l}\text { Categoria } \\
\text { de MRC }\end{array}$} & \multicolumn{4}{|l|}{ Admissão } & \multicolumn{2}{l|}{ Alta } & \multirow{2}{*}{${ }^{*}$} \\
\cline { 2 - 5 } & $N$ & $\%$ & $N$ & $\%$ & \\
\hline Normal & 23 & 71,9 & 25 & 78,1 & 0,62 \\
\hline Fraqueza & 9 & 28,1 & 7 & 21,9 & \\
\hline
\end{tabular}

N: número; \%: porcentagem

*Adotando $p<0,05$ para diferença estatística, com teste de McNemar.

Fonte: Dados da pesquisa.

Na Tabela 5, podemos observar os valores obtidos por meio das variáveis do Índice de Barthel. Conforme os resultados apresentados, houve diferença significativa entre a admissão e a alta hospitalar para o índice de atividades rotineiras, onde o valor apresentado na alta foi menor que $o$ apresentado na admissão $(p=0,04)$. A diferença para o Índice de Barthel geral não foi significativa. 
Tabela 5 - Comparação antes e depois do Índice de Barthel, quanto aos domínios

\begin{tabular}{|l|l|l|l|l|l|l|l|}
\hline \multirow{2}{*}{ Variável } & \multicolumn{2}{l}{ Admissão } & \multicolumn{2}{l|}{ Alta } & $p^{*}$ \\
\cline { 2 - 9 } & Mediana & Média & Mín-Máx & Mediana & Média & Mín-Máx & \\
\hline Índice de Barthel & 97,5 & 83,4 & $20-100$ & 85 & 78,6 & $20-100$ & 0,07 \\
\hline Alimentação & 10 & 9,0 & $5-10$ & 10 & 8,9 & $0-10$ & 0,65 \\
\hline Banho & 5 & 3,7 & $0-10$ & 5 & 3,2 & $0-10$ & 0,18 \\
\hline Atividades Rotineiras & 5 & 4,2 & $0-5$ & 5 & 3,6 & $0-5$ & 0,04 \\
\hline Vestir-se & 10 & 8,6 & $5-10$ & 10 & 8,2 & $5-10$ & 0,15 \\
\hline Intestino & 10 & 8,9 & $0-10$ & 10 & 9,0 & $0-10$ & 0,31 \\
\hline Sistema Urinário & 10 & 7,9 & $0-10$ & 10 & 7,6 & $0-10$ & 0,48 \\
\hline Uso de toalete & 10 & 7,9 & $0-10$ & 10 & 7,5 & $0-10$ & 0,18 \\
\hline Transferência & 15 & 12,0 & $5-15$ & 12,5 & 11,2 & $5-15$ & 0,16 \\
\hline Mobilidade & 15 & 13,4 & $5-15$ & 15 & 12,6 & $5-15$ & 0,06 \\
\hline Escadas & 10 & 7,3 & $0-10$ & 10 & 7,0 & $0-10$ & 0,31 \\
\hline
\end{tabular}

Mín: mínimo; Máx: máximo,

*Adotando $p<0,05$ para diferença estatística, com teste de Wilcoxon.

Fonte: Dados da pesquisa

De acordo com a Tabela 6, a maioria dos pacientes foi classificada como leve e independente no momento de admissão na UTI. Entretanto, na alta da UTI para a enfermaria houve distribuição de alguns pacientes para moderado e leve.

Tabela 6 - Comparação antes e depois do Índice de Barthel, quanto à classificação da funcionalidade

\begin{tabular}{|l|l|l|l|l|l|}
\hline \multirow{2}{*}{ Classificação do Barthel } & \multicolumn{2}{l|}{ Admissão } & \multicolumn{2}{l|}{ Alta } & \multirow{2}{*}{$p^{*}$} \\
\cline { 2 - 7 } & $N$ & $\%$ & $N$ & $\%$ & \\
\hline Independente & 16 & 50,0 & 12 & 37,5 & 0,06 \\
\hline Leve & 12 & 37,5 & 13 & 40,6 & \\
\hline Grave & 2 & 6,3 & 2 & 6,3 & \\
\hline Moderada & 2 & 6,3 & 5 & 15,6 & \\
\hline
\end{tabular}

N: número; \%: porcentagem.

*Adotando $p<0,05$ para diferença estatística, com o teste de Wilcoxon.

Fonte: Dados da pesquisa. 


\section{DISCUSSÃO}

0 presente estudo teve o sexo masculino como maior parte da população avaliada, resultado semeIhante ao encontrado em diversos estudos, como por Blanes e outros autores (2004), Oliveira e colaboradores (2010), Moulim e outros autores (2012) e Cordeiro e colaboradores (2015), esses dados podem ser explicados pelo fato de mulheres procurarem atendimento de saúde com mais frequência que os homens, evitando assim o agravo dos problemas, contrariando o estudo de Carvalho e outros autores (2013), que encontraram um maior número de mulheres internadas.

Outro fator avaliado foi a idade média da amostra, encontrando valor de 54,4 anos, resultado semelhante ao de Mello e Fortes (2010), que encontraram uma média de idade de 52,81 anos. Martinez e outros autores (2013), encontraram um valor maior, mas ainda próximo, com média de 57,5 anos. Outro dado encontrado foi o tempo de internação, com uma média de 5,3 dias, condizente ao relatado por Dietrich e outros autores (2014) de 5,81 dias, na pesquisa de Abelha e colaboradores (2006) a média foi de 4,09 dias, divergindo outro artigo que apresenta um valor maior, com 9,7 dias de internação (MOULIM et al., 2012).

No atual estudo, foram diversos os motivos da internação na UTI, porém doença renal aparece em 34\% dos casos, seguido de doenças cardiovasculares e infecções com $15 \%$ cada. No estudo de Feliciano e outros autores (2012), 54\% da amostra esteve internada na UTI devido o diagnóstico de insuficiência respiratória aguda, já outros estudos apresentaram prevalência de cardiopatias como motivo da admissão (PASINI et al., 1996; DIETRICH et al., 2014).

No presente estudo, a avaliação prospectiva realizada em 32 pacientes admitidos na UTI, não identificou diferença significativa no resultado da força muscular periférica apresentada de acordo com o teste aplicado, quando comparado os valores encontrados no momento da admissão e da alta da unidade.

De acordo com o resultado da pesquisa, pode-se ter a impressão de que o internamento na UTI não acarretou em declínio da força muscular periférica, tendo em vista que não houve variação significativa do MRC. Porém al- guns estudos realizados de forma semelhante encontraram resultados diferentes, quando realizado o MRC em pacientes internados que fizeram uso de ventilação mecânica, ao fim da pesquisa foi notória a redução da força motora global dos pacientes, mesmo quando realizado o despertar diário e à mobilização precoce (RODRIGUES et al., 2010; DANTAS et al., 2012; LOUREIRO et al., 2012). Esta divergência nos resultados sugere que o processo de intubação representa um grande marco na diminuição da força muscular dos pacientes na UTI.

Segundo Costa e outros autores (2014) pacientes hospitalizados em enfermarias, não apresentam alteração de independência funcional, diferente dos pacientes internados em uma UTI de acordo com a pesquisa atual, onde a população estudada apresentou um declínio em todos os âmbitos pesquisados, porém apenas atividades rotineiras teve uma redução significativa, corroborando com o resultado de Mafra (2012). Entretanto, Barata e Silva (2012), encontraram um resultado diferente, em seu estudo, ao utilizar o Barthel percebeu que a funcionalidade do paciente aumentou quando comparado ao momento da admissão com o momento da alta da UTI.

Outros estudos utilizaram o Medida da Independência Funcional (MIF), para a avaliação da funcionalidade, onde foi observada redução significativa da independência funcional dos pacientes avaliados (GARCIA et al., 2012; MARTINEZ et al., 2013). Em contrapartida o resultado encontrado por Mello e Fortes (2010) difere dos anteriormente citados, havendo uma melhora da funcionalidade desde o momento da admissão até a alta.

\section{CONCLUSÃO}

Com base no estudo apresentado, conclui-se que apesar do tempo de internação dos pacientes na UTI, não houve perda significativa da força muscular periférica, entretanto, no Índice de Barthel, o resultado encontrado na alta para "Atividades rotineiras", foi menor significativamente que o resultado apresentado na admissão. Este resultado mostra que apesar do sucesso do protocolo adotado pela equipe, ainda houve redução significativa da funcionalidade. 


\section{REFERÊNCIAS}

ABELHA, F.J. et al. Mortalidade e o Tempo de Internação em uma Unidade de Terapia Intensiva Cirúrgica. Rev. Bras. Anestesiol., Rio de Janeiro, v.56, n.1, p.34-45, 2006.

AZEREDO, C. Fisioterapia respiratória. Rio de Janeiro: Panamed, 1984.

BARATA, V.; SILVA, W. Avaliação da capacidade funcional para atividades da vida diária em pacientes internados na unidade de terapia intensiva. Rev.

Bras. Fisioterapia, São Carlos, v.16, p.214-214, 2012.

BLANES, L. et al. Avaliação clínica e epidemiológica das úlceras por pressão em pacientes internados no hospital São Paulo. Rev. Assoc. Med. Bras., São Paulo, v.50, n.2, p.182-187, 2004.

BUHOP; K. Pulmonary rehabilitation in the intensive care unit. In: Fishman AP, (Ed). Pulmonary rehabilitation. New York: Marcel \& Dekker, p.725-738, 1996.

CARVALHO, T. et al. Relação entre saída precoce do leito na unidade de terapia intensiva e funcionalidade pósalta: um estudo piloto. Rev. Epidemiol. Control. Infec., Santa Cruz, v.3, n.3, p.82-86, 2013.

CIESLA, N. Chest physical therapy for patients in the intensive care unit. Phys Ther., Virginia, v.76, n.6, p.609-25, 1996.

CORDEIRO A. et al. Análise do grau de independência funcional pré e na alta da UTI em pacientes submetidos à cirurgia cardíaca. Rev. Pesq. Fisioter., Salvador, v.5, n.1, p.21-27, 2015.

COSTA, F. et al. Avaliação da funcionalidade motora em pacientes com tempo prolongado de internação hospitalar. UNOPAR Cient., Ciênc. Biol. Saude, Londrina, v.16, n.2, p.87-91, 2014.
DANTAS C. et al. Influência da mobilização precoce na força muscular periférica e respiratória em pacientes críticos. Rev. Bras. Ter. Intensiva, São Paulo, v.24, n.2, p.173-178, 2012.

DE JONGHE, B. et al. Respiratory weakness is associated with limb weakness and delayed weaning in critical illness. Crit. Care Med., New York, v.35, n.9, p.2007-2015, 2007.

DESAI, S.; LAW, T.; NEEDHAM, D. Long-term complications of critical care. Crit. Care Med., New York, v.39, n.2, p.371-379, 2011.

DIETRICH, C. et al. Funcionalidade e qualidade de vida de pacientes internados na Unidade de Terapia Intensiva. ASSOBRAFIR Ciência, Londrina, v.5, n.1, p.41-51, 2014.

FELICIANO, V. et al. A influência da mobilização precoce no tempo de internamento na Unidade de Terapia Intensiva. ASSOBRAFIR Ciência, Londrina, v.3, n.2, p.31-42, 2012.

GARCIA N.G. et al. Avaliação da independência funcional de pacientes pós-internados em unidade de terapia intensiva. ConScientiae Saúde, São Paulo, v.11, n.2, p.296-304, 2012.

KUWABARA, K. et al. Associations between the use of critical care procedures and change in functional status at discharge. J. Intensive Care Med., Thousands Oaks, v.28, n.5, p.296-306, 2013.

LOUREIRO, T. et al. Utilização da escala mrc para avaliação funcional do paciente crítico após extubação. Rev. Bras. Fisioter., São Carlos, v.16, supl., p.455-455, 2012.

\section{MAFRA, J. Avaliação da qualidade de vida e funcionalidade do paciente crítico após alta hospitalar. 2012. 107f. Dissertação (Mestrado) - Universidade de São Paulo, São Paulo, 2012.}


MALKOÇ, M.; KARADIBAK, D.; YILDIRIM, Y. The effect of physiotherapy on ventilator dependency and the length of stay in an intensive care unit. Int. J. Rehabil. Res., Rheinstetten, v.32, n.1, p.85-88, 2009.

MARTINEZ, B. et al. Declínio funcional em uma unidade de terapia intensiva (UTI). Rev. Inspirar, Curitiba, v.5, n.1, p.1-5, 2013.

MCDOWELL, I.; NEWELL, C. Measuring health: a guide to rating scales and questionnaires. 2 nd ed. New York: Oxford University Press; 1996.

MELLO, C.; FORTES, M. Perfil funcional de pacientes neurológicos internados em UTI. Rev. Bras. Fisioter. São Carlos, v.14, supl.2, p.1-11, 2010.

MOULIM, M. et al. Comparação da funcionalidade no momento da alta de pacientes internados em uma UTI clínica e a outra cirúrgica: estudo prospectivo observacional. Rev. Bras. Fisioter., São Carlos, v.16, supl., p.425-425, 2012.

OLIVEIRA, A. et al. Fatores associados à maior mortalidade e tempo de internação prolongado em uma unidade de terapia intensiva de adultos. Rev.

Bras. Ter. Intensiva., São Paulo, v.22, n.3, p.250-256, 2010.

PASINI, D. et al. Diagnósticos de enfermagem de pacientes internados em unidades de terapia intensiva (UTI). Rev. Esc. Enferm. USP., São Paulo, v.30, n.3, p.501-518, 1996.
RIVOREDO, M.G.A.C.; MEIJA, D. A cinesioterapia motora como prevenção da síndrome da imobilidade prolongada em pacientes internados em Unidade de Terapia Intensiva. 2013. Disponível em: <www. portalbiocursos.com.br/artigos/fisio_intensiva/03. pdf >. Acesso em: 7 jan. 2017.

RODRIGUES, I. et al. Fraqueza muscular adquirida na unidade de terapia intensiva: um estudo de coorte.

Rev. Bras. Cienc. Saúde, João Pessoa, v.8, n.24, p.815, 2010.

SACANELLA, E. et al. Functional status and quality of life 12 months after discharge from a medical ICU in healthy elderly patients: a prospective observational study. Crit Care, London, v.15, n.2, R105, 2011.

SIBINELLI, M. et al. Efeito imediato do ortostatismo em pacientes internados na unidade de terapia intensiva de adultos. Rev. Bras. Terap. Intensiva, São Paulo, v.24, n.1, p.64-70, 2012.

SILVA A.P.P.; MAYNARD, K.; CRUZ, M.R. Efeitos da fisioterapia motora em pacientes críticos: revisão de literatura. Rev. Bras. Terap. Intensiva, São Paulo, v.22, n.1, p.85-91, 2010.

STILLER, K. Physiotherapy in intensive care: towards and evidence-based practice. Chest, Chicago, v.118, n.6, p.1801-1813, 2000. 
Recebido em: 13 de Novembro de 2016 Avaliado em: 16 de Novembro de 2016 Aceito em: 28 de Novembro de 2016

\begin{abstract}
1 Programa de Pós-graduação em Química e Biotecnologia da Universidade Federal de Alagoas - UFAL, Maceió, AL. Email: carolina_ calles@hotmail.com
\end{abstract}

2 Graduado em Fisioterapia pelo Centro Universitário Tiradentes, MaceióAL.Email: junior_psc@hotmail.com

3 Graduado em Fisioterapia pelo Centro Universitário Tiradentes, MaceióAL. Email: camilamenezes.fisio@hotmail.com

4 Especialista em Fisioterapia em Terapia Intensiva. Faculdade Estácio de Alagoas, Maceió-AL. Email: evelin-fisio@hotmail.com

5 Especialista em Terapia Intensiva. Faculdade da Cidade de Maceió FACIMA, Maceió-AL. Email: larascamilo@hotmail.com 